\title{
LES FOUILLES DU PÈGUE (Drôme) de 1957 à 1975
}

Deuxième Partie

par Jean-Jacques HATT

III. Les resles du grenier hallslallien (fig. 26).

La plupart du temps, les vestiges du grenier hallstattien ne sont pas en place. Ils ont été remployés pour servir de matériaux de remblai, principalement à deux reprises : au $\mathrm{N}^{\mathrm{e}}$ siècke, lors de l'installation dans les ruines de l'oppidum d'ateliers de potiers, et à la fin du rve siècle, lors des profonds remaniements exécutés en vue de la construction d'une nouvelle ville fortifiée reprenant l'ancien emplacement. (ce n'est qu'en de rares endroits, miraculeusement préservés, que des vestiges cohérents ont pu être mis au jour in silu.

Au sondage 7, en $x=26 \mathrm{~m}$ à $31 \mathrm{~m}, \mathrm{y}=2 \mathrm{~m}$ à $7 \mathrm{~m}$, dans les carrés 56,66 , nous arons pu dégager et étudier une partie du grenier assez bien conservée. Il s'agit d'une logette de 5 m de longueur sur $3 \mathrm{~m}$ de largeur, installée dans la partie surl-est de la grande maison $\mathrm{A}$, après en avoir éliminé les dalles et creusé le sol. Lne banquette assez irrégulière, en argile sur base de pierres sèches, large de $1 \mathrm{~m}$, avait été aménagée le long du mur de terrasse supérieur, et perpendiculairement à celui-ci, le long de la rangée de pierres qui servait de fondation à la paroi nord-est de la logette, une seconde banquette large de $0,75 \mathrm{~m}$. En $\mathrm{x}=$ $30,20 \mathrm{~m}, \mathrm{y}=6 \mathrm{~m}$ à $6,50 \mathrm{~m}$, ont été mis au jour des restes de poutre et de planche carbonisée reposant sur un double muret de pierres sèches construit à l'intérieur de la maison hallstattienne, dans la même orientation qu'elle (fig. :26). A plusieurs reprises ont été observés des restes de pisé calciné portant la trace d'entrelar's de branchages et mème certains vestiges de branchages entrelaces carbonisés. Six fonds de jarres, autant de vases de moyennes dimensions entiers, presque entiers ou reconstituables ont été trouvés in silu. On peut se représenter aisément ce type de logette, correspondant en surface aux plus petites cabanes hallstattiennes, d'environ 15 a $20 \mathrm{~m}^{2}$. Elles étaient constituées d'un assemblage de murs en torchis armés d'un entrelacs de branchages, reposant sur des fondations irrégulières de murettes en pierres sèches installées dans les ruines des habitations hallstattiennes. A l'intérieur de ces cases avaient été installées des banquettes en argile destinées à supporter 


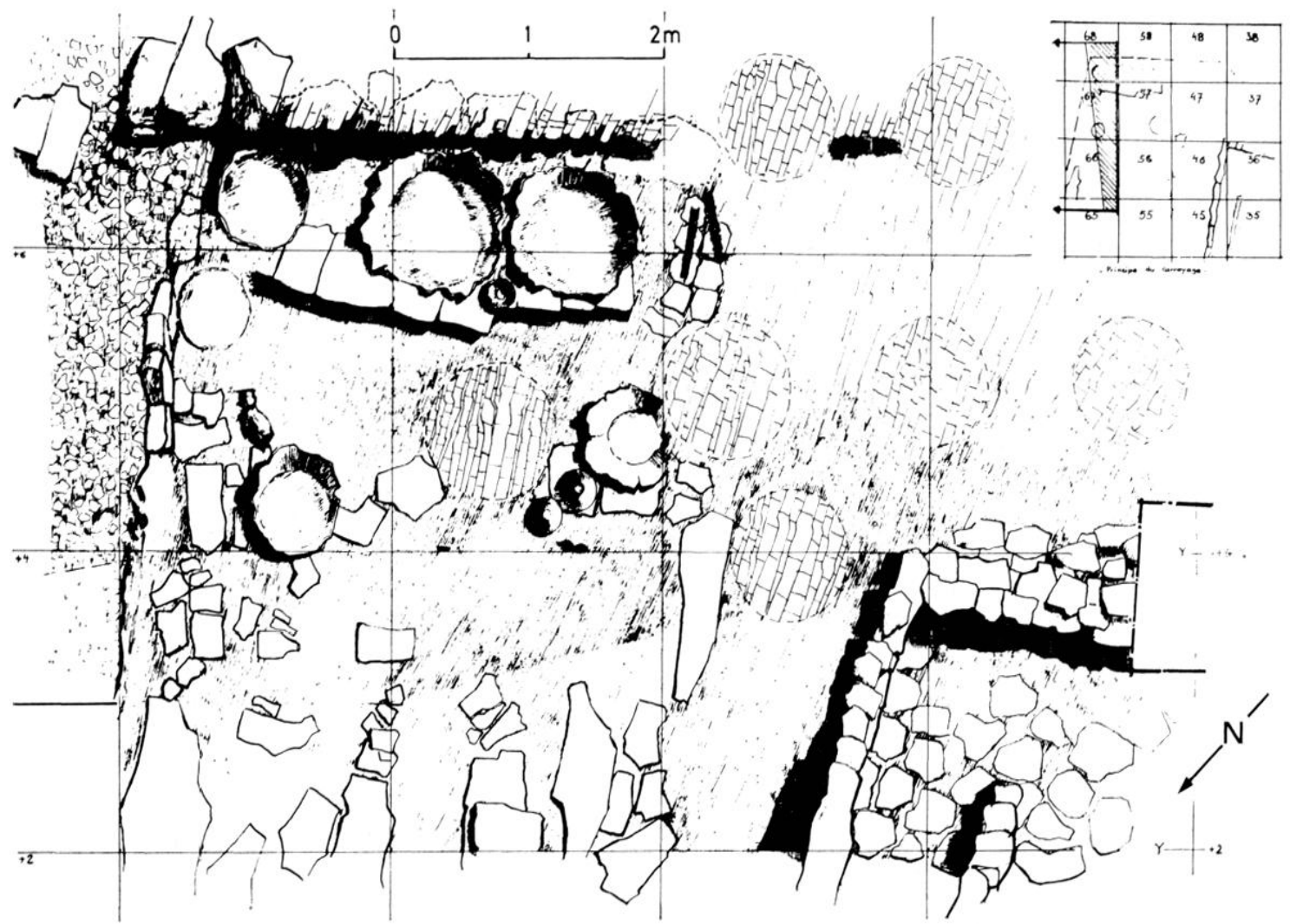

iff Sondage 7 , plan dibiallé de la logette du grenier.
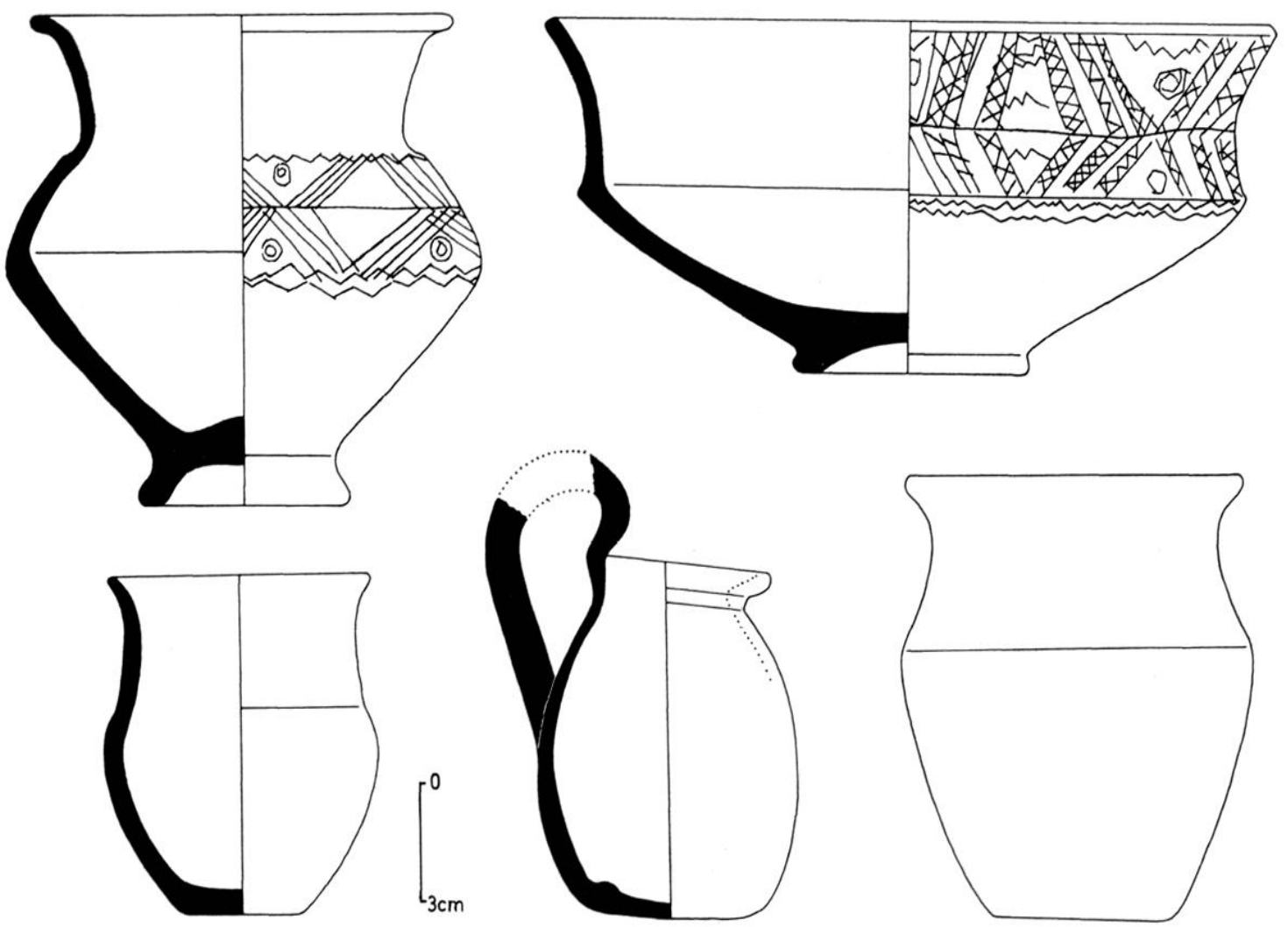

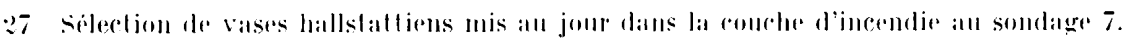


des jarres en torchis modelées et séchées sur place. Yous avons vu plus haut que ce dispositif avait déja éte pratique pour conserver des provisions all rez-de-rehaussé de la tour interieure.

Le nombre et la dimension des vases retrouves sur place posent un problème. En effet, il y avait dans les intervalles des jarres remplies de grains. des vases de grande et de moyenne dimension remplis de grains et apportés là de toute évidene postérieurement a l'installation des jarres of all storkage des grains dans cess dernières. Il y arait également de petits vases, gobelets ou coupes trouvés parfois a l'intépieur des vases plus importants. ou à ròté d'eux (fig. 27). ('es rlerniers semblent bien avoir été utilisés pour la manipulation des grains. Il parait difficile d'expliquer cette abondance de recipients autrement que par l'hypothese suivante. que je dois à mon ami Ch. Lagrand : en dernière minute, devant l'imminence de l'invasion et dans l'affolement général, toute la population avait rassemblé la vaisselle disponible, rempli ces vases de grains, et tous ansemble, hommes, femmes, enfants, avaient alors porté chacun son fardeau de grains sur la colline. C'est ainsi que la rouche d'incendie du Pègue contient véritablement toute la vaisselle d'une génération d'indigènes vers 480 av. J.-C. Nomettons point les murs obliques de la maison B dans les carrés 34, 43, 44, 45, qui appartiennent aux remaniements du début du ve siècle. En prévision du grenier to sol de cette habitation avait été d'autre part surcreusé, et la logette installée en contrebas. Malheureusement la couche d'incendie dans ces parages, pour épaisse qu'elle soit, a été totalement bouleversée.

\section{Chanlier central.}

L'aménagement du grenier dans le chantier rentral était branché sur le mur de fondation hallstattion doublant le mur de terrasse. La logette s'appuyait d'une part sur le mur hallstattien remanié et remployé, et d'autre part sur une file de pierres plates située à deux metres alu sud-ouest de colle-ci. Cette file de pierres servait de base à une ligne de poutres supportant le toit de la logette. Elle ne constituait que la partie centrale de l'ensemble, continuant vers te surb-ouest. jusqu'à une limite qui n'a pas éte conservée. Je pense que la logrette présentait une longueur de $4 \mathrm{~m}$ du surl-ouest au nord-est et une largeur de $2,50 \mathrm{~m}$ du nord-ouest all sud-est. Quelques vases indigènes at un grand vase en céramique pseudo-ionienne reconstituables ont été mis all jour dians rette logette. Ils rontenaient tantòt du grain, tantôt des vesces carbonisés.

\section{Greniers hallstalliens consereres an sondage 11.}

Au-dessus du sol El de la tour intérieure avait été aménagé un fort remblai en argile à peu près stérile de $0,80 \mathrm{~m}$ l'épaisseur, qui contenait uniquement une amphore massaliote du ve siècle al. J.-(i. sans col ni anse. Dans ce remblai avait été creusé une rigole de drainage de profil triangulaire qui fut remplie en partie par les restes de l'incendie du grenier. Le reste de la couche d'incendie avait totalement disparu, sauf en un endroit, au-delà de la rigole, au-dessus de la cour darcueil, mais aucune jarre ne fut trouvée en place. 


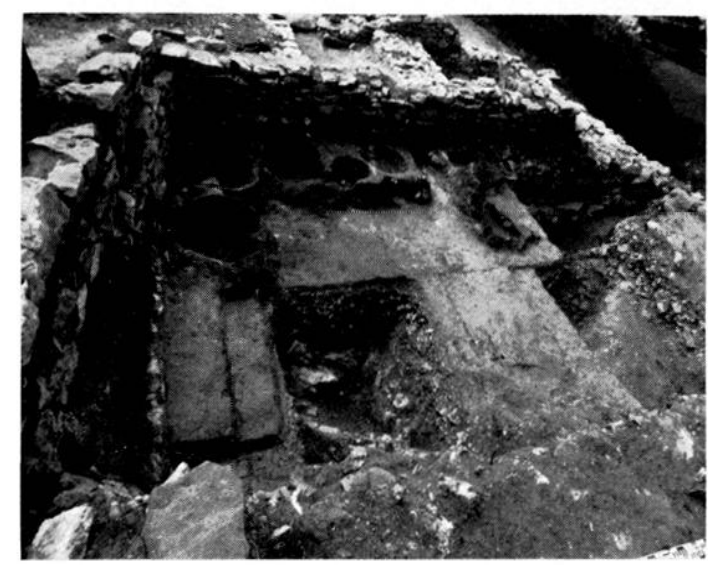

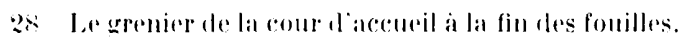

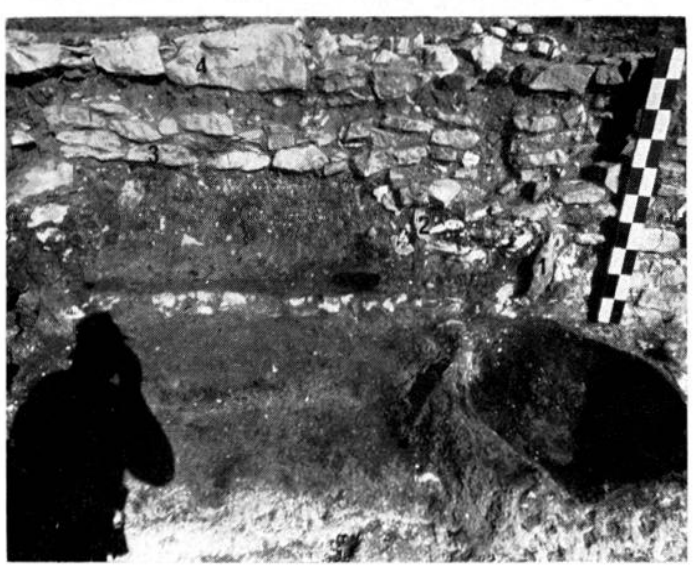

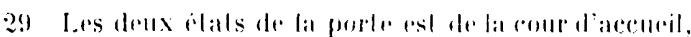
of les murs de lat Tome; 1, premier blal ; ?, deuxieme

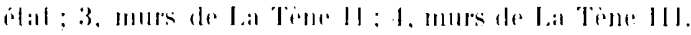

La cour d'arecueil a ete dans son ensemble aménagere elle-mème an grenier. A refle fin, un mur a ete construit sur la face nord-ouest. une banquette en argile amenagese sur tout le pourtour, la porte nord fut hourhè et l'entrée sud fut rétrécie afin de laissere plus de place aux jarres en torehis. Dex fonds de jarres ont ete retrouves en placere, la plupart vidées

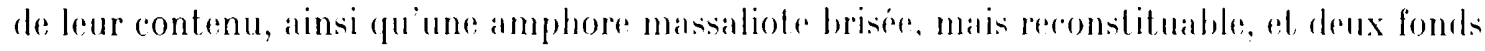
de caisses egalement on torehis. De nombreux vases indigenes, brises sur place certains d'assez grandes dimensions, ont pu itre reconstitués. Malheurensement ce grenier hallstattien arait ete en grande partie boulererse par l’installation des ateliers céramigues du $\mathrm{r}^{\mathrm{e}}$ siècle (fig. $\left.2 \mathrm{x}-2 !\right)$ ).

lans le couloir d'entrée prolongeant la porte monument ale a che mis an jour, en 19731974, dans la partie nord-ouest du chant ier (carrés 333.1.335.), 3336) un mur du grenier. oriente nord-ouest suderst, a lointerieur duquel se trouvait un lambeau de courehe lol an place, mais malheureusement aucune jarre in silu. Ce mur part a son extremite norel-ouest deun vestige de mur de terrasse hallstattien. Il repose, soit directement sur er mur, soit sur un cailloutis dame.

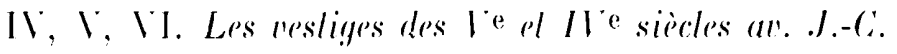

Les fouilles ont demontre, particulierement all sondage 8 at an somblage 11, quil !

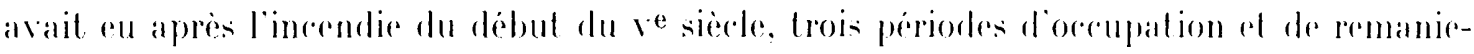
ments: une phase d'installations assez inorganiques dans le courant du vesiede. un établissement plus durable et plus st ructure consistant surtout en ateliers art isanaux potiers et peut-ètre métallurgistes) au cours du we siècle. une reconstruction de lenceinte et de profonds bouleversements du systeme de terrasses a la fin du re siede. Cette derniere période ne semble pas d'ailleurs avoir été suivie diune longue orcupation jusqu'a la fin du

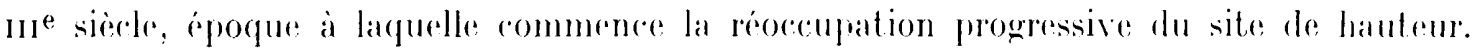




\section{Le sondage 10.}

La fouille duS. 7 n'a pas mis au jour de restes très importants ni très cohérents de ces trois périodes, si ce n'est les modifications dans le tracé des murs dans la région nordouest, et la juxtaposition de deux terrasses postérieures à l'incendie dans la zone sud-ouest.

lin ce qui concerne la plus ancienne périorle de réoccupation, ve siècle av. J.-C., une analyse des restes du grenier nous a permis de constater qu'il y avait eu des modifications dans les murs de la logette de ce dernier, dans sa partie nord-est. Les pierres avaient été déplacées pour ètre remises (les parties calcinées des pierres n'étaient plus à leur place normale, et certaines d'entre elles reposaient sur la couche d'incendie). On pouvait également remarquer qu'il y avait une interruption dans la ligne des jarres. Il s'agit là d'une réoccupation des ruines pour y installer des habitats de fortune. D'autres indices du même genre ont bé observés au S. 8 et au S. 11.

lans la partie sud-ouest du chantier, il existe deux systèmes de terrasses postérieures à l'incendie. L'un prend son départ entre le mur de Lerrasse hallstattien médian et la banquette du grenier aménagée dans la maison $\mathrm{B}(\mathrm{x}=34,50 ; \mathrm{y}=3,75)$ à $4 \mathrm{~m})$. Il se poursuit vers le nord-est, puis se perd dans les carrés 84, 85. I'après son étage stratigraphique, il appartient au rve siècle. Il est daté par d'assez nombreuses trouvailles de céramique attique a figures rouges, la plupart rassemblées dans les parages des terrasses bouleversées et reconstruites. L'autre est légèrement décalé à son départ par rapport au mur hallstattien qu'il domine, puis se dirige vers le sud-est. Il aboutit dans les carrés 104, $114,124,134$, a des restes de terrasses bouleversées dominés par de grandes dalles. Dans les carrés 11;) et 125 une amphore massaliote culbutée à laquelle manquait le fond marquait l'emplacement, d'une habitation de la fin du ave siècle ayant utilisé les gros blocs et les dalles des installations hallstattiennes.

La coupe stratigraphique dans les carrés 66 et 67 au sud-est du mur hallstattien médian, a fait apparaître une zone de perturbation qui se manifeste par la présence de pierres plates plantées verticalement ou disposées obliquement. Ces pierres plates sont incluses dans un remblai dominant les couches en place, qu'il s'agisse de D1 à l'emplacement de la logette du grenier, ou de E dans la région située plus au nord-est. Dans les carrés 75, 85, 95, ont été trouvés de nombreux fragments de céramique attique à figures rouges du ive siècle, particulièrement concentrés le long des murs de terrasse médians. C'est là sans aucun doute le résultat des remblais du début et de la fin du $v^{\text {e }}$ siècle qui ont eu pour résultat d'accumuler les matériaux aux points de rupture de la pente, le mème fait a été observé au S. 11 en particulier dans le couloir prolongeant la porte monumentale.

Jans cette zone ont été trourées une fibule en bronze et une agrafe en fer hallstattiennes, sans que malheureusement ces objets aient une valeur de datation pour les couches qui les contenaient. Par contre la couche de remblai d'incendie ('2 a livré dans le carré 124, en $\mathrm{x}=14,15 \mathrm{~m}, \mathrm{y}=0,20 \mathrm{~m}$ une fibule gauloise de La Tène Ilb qui contribue, avec les tessons à figures rouges, a dater la couche et le mur qui lui correspond (fig. 30).

N'ayons garde d'omettre les remaniements qui, au rve siècle, ont modifié légèrement le tracé de la maison $E$ de telle façon que le plan primitif est aisément reconnaissable. Toutefois les murs actuels reposent en grande partie sur la couche d'incendie, et le sol qui leur correspond est daté par de la céramique attique à figures rouges du re siècle. 


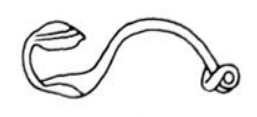

| 1

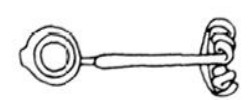

0
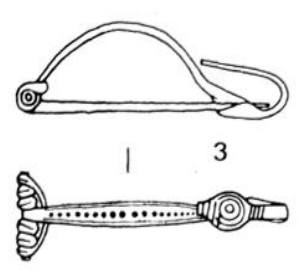

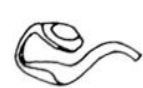

12

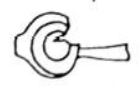

$2 \mathrm{~cm}$

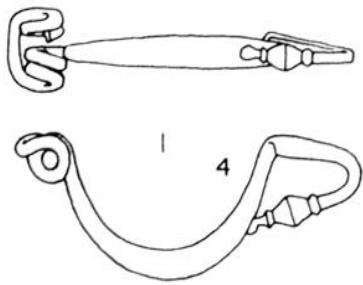

30 Jihules decourertes dans les conches du remblai du W" siecle all sondage 7 : 1 et : fibuies hallstalliennes: 3 , fibule de transition Ilallstall-Ja Tene: 1, tibule du début de Ia t'ine lb.

\section{Le chanlier central.}

Le mur de terrasse mal conservé oblique par rapport aux murs de terrasse hallstattiens,

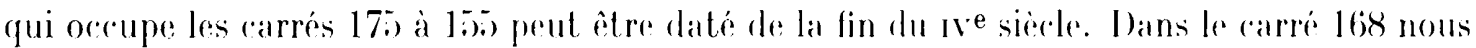

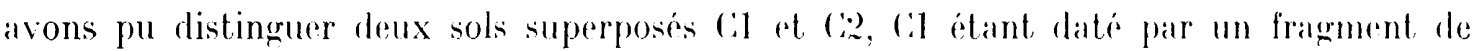
céramique pré-Ginathia représentant une danseuse, dont le milieu du corps at le bras gatuche sont seuls conservés (fig. 31).

\section{Le sondage 11.}

\section{La lour intériéure.}

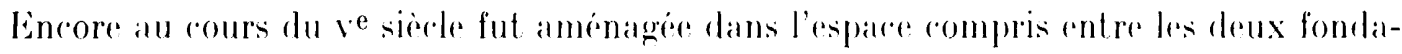
tions de lessalier de la tour intérieure une habitation de fortune. En effet. le sol fut alors sommairement aplani par la pose de quelques pierres plates dominant, le cailloutis hallstattien. La couche noire dhabitat rorrespondant a ce sol contenait un ressort ed une aiguille de fibule de La Tène la ainsi qu'un rebord d'assiette de même époque.

Au cours du re sière la rigole du grenier et la terrasse en remblai qui dominaient ae dernier furent coupées par une tranchée destiné à donner aces à une demeure installée dans les ruines, à l'intérieur de la tour, dont le remplissage avait eté surcreusé, libérant la base du mur de terrasse hallstattien médian. En effet, le long de ce mur avaient dete

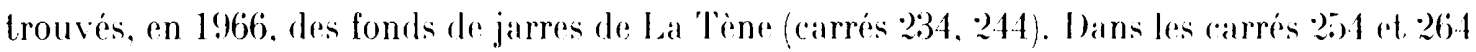
devaient être découverts dans la suite deux chenets gravés en terre cuite faisant la paire. l'un entièrement ronserve a lexeeption de son ornement terminal (tète de bélier). l'autre seulement à moitié (fig. 32). Des tessons de céramique attique à figures rouges du roe siocele fournissaient la date de cette installation (jig. 33). In col d'amphore massaliote de cette époque fut également mis au jour dans le carré 24.3. Il etait renversie et posésur la fondation médiane du bastion. La tranchée du rve siecle dans la partie nord-est du bastion, a été remplie à la fin du ro siècle par un remblai affectant l'aspect d'un éboulis. Ca dernier longeait le mur sud-ouest ret vers le sud-ouest atteignait le substrat qui avait ete particlle- 


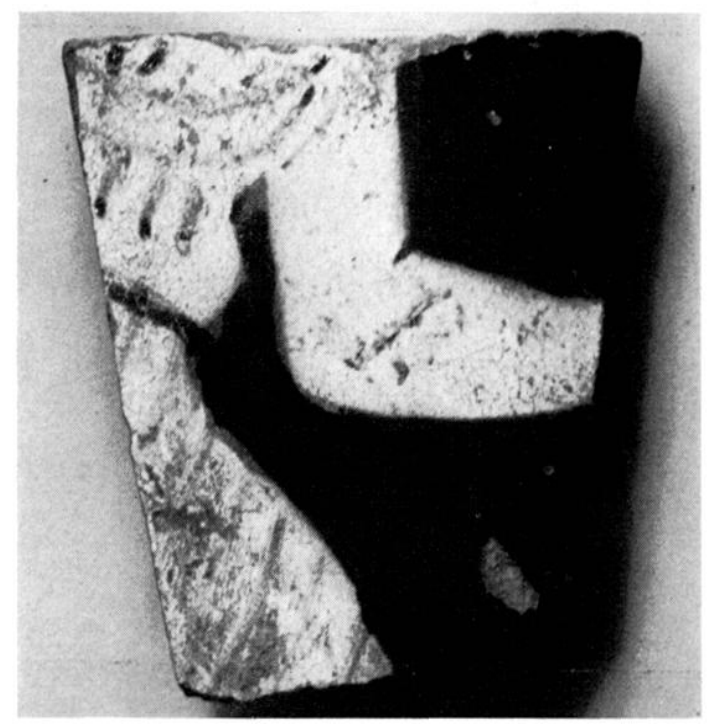

31 Cépamique priefintalluia

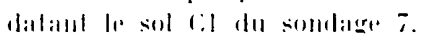
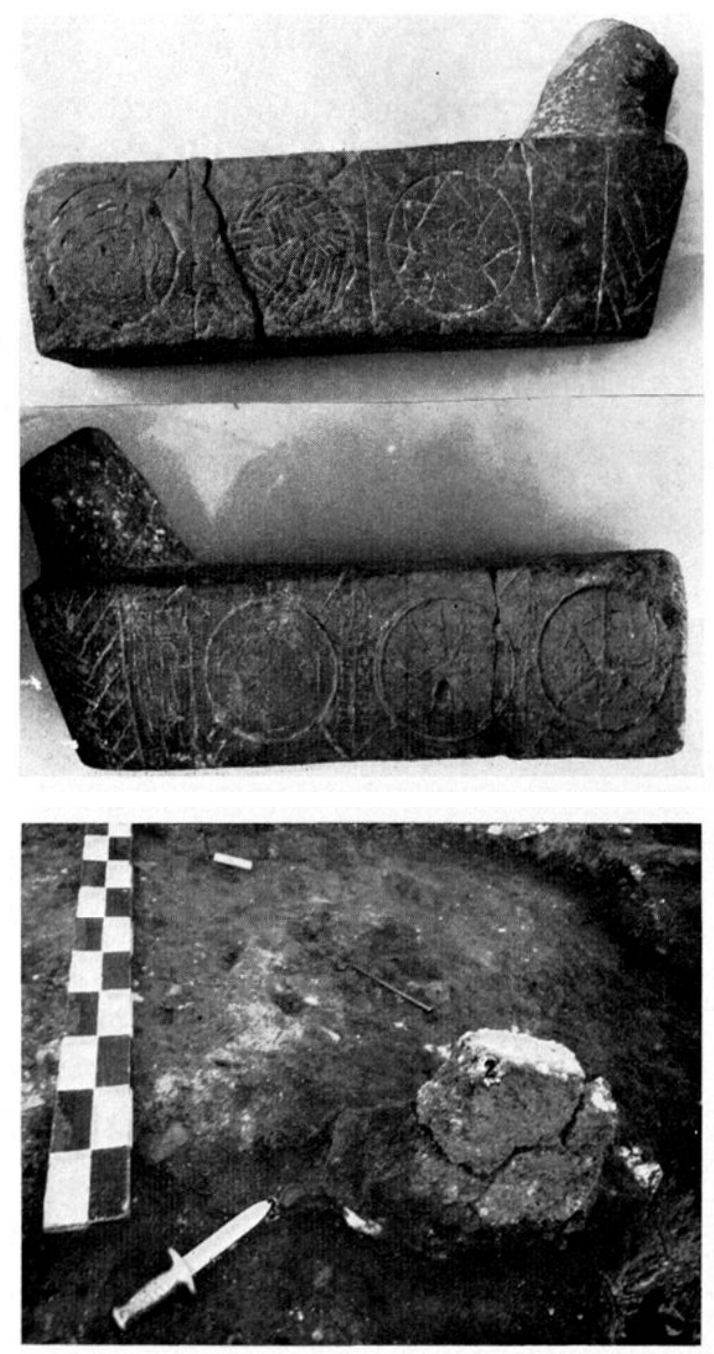

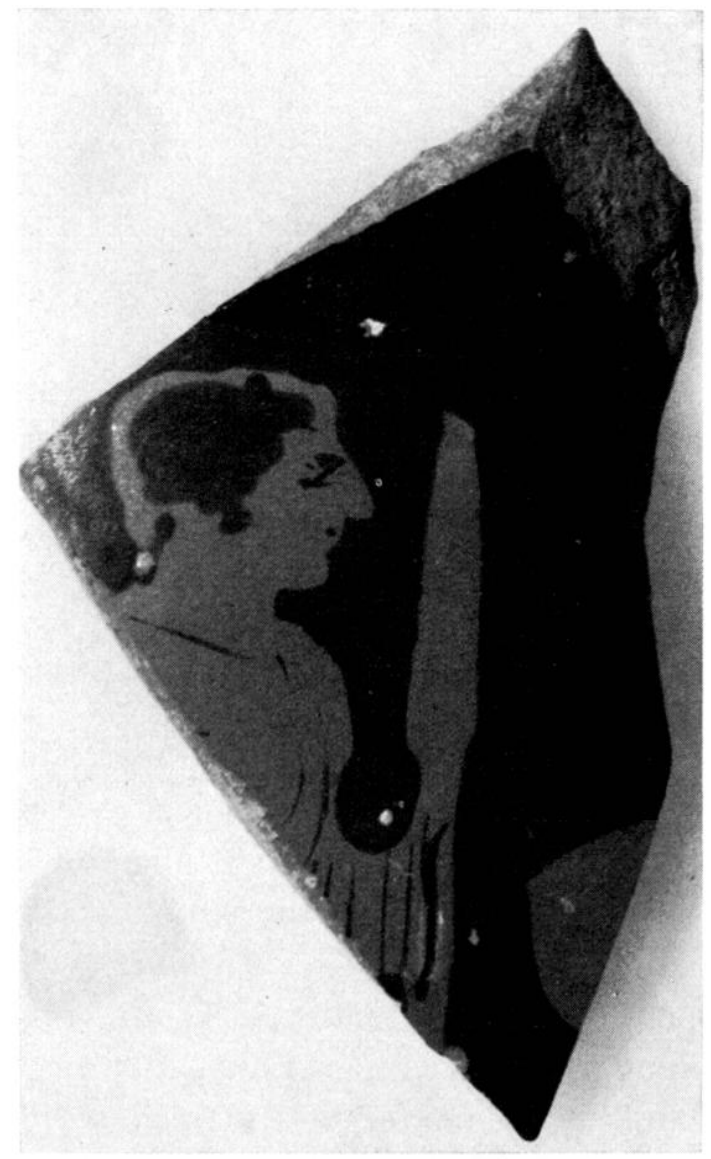

33 Tesson atlique à figures rouges,

trouve dans la cave de cabane de la lour.
3: Chemel rotif gaulois de la periode de la tène lb, trouse dans ba cave de la calane amenagee a l'intérieur de lat tour.

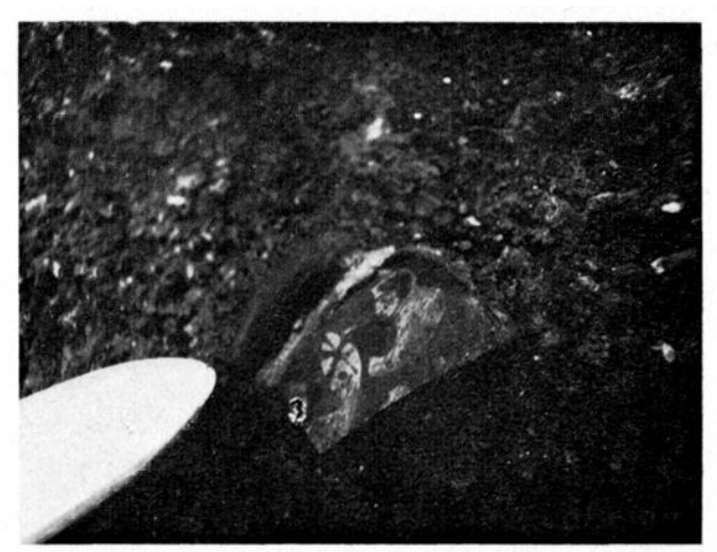

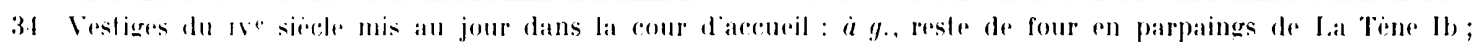

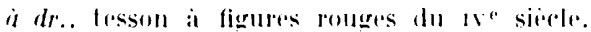


ment mis à nu par les terrassements du $\mathrm{N}^{\mathrm{e}}$ siècle. Ces remblais s'étaient faits à cheval sur les fondations de la tour intérieure et de la cour d'accueil voisine, et un noureau nivellement avait été réalisé entre les deux emplacements. A ce niveau bl correspond un lit régulier de pierres plates. Au-dessous de ce niveau ont été trouvés des tessons a figures rouges du re siècle. Nulle part ailleurs nous n'avons trouve les courhes $(i 1$ it $C 2$ aussi faciles i distinguer.

Après l'incendie du début du ve siècle qui anéantit le grenier, la cour d'accueil fut abandonnée longtemps, puis, al cours du re sièce, convertie an atelier de potiers. Ieux groupes de fours doivent ètre distingués : contre l'entrée un tas de pierres rougies par le feu et de parpaings calcinés, de $3 \mathrm{~m}$ de diamètre environ, était constitué entièrement de restes de fours. Ces derniers araient utilisé la porte comme cheminée, et les pierres des murs de cette derniere el du couloir situé en face avaient eté rougies par la chaleur (carrés 276, 286). I'autre part, dans le carré 28:3 ont été dégagés les restes d'un four en parpaings d'argile cuite mêlé de cailloux (fig. 3.1). Le fragment du four epais de $0,12 \mathrm{~m}$ présentait une hauteur de $0,32 \mathrm{~m}$ et une longueur de $0,45 \mathrm{~m}$. Le diamet re de ce four peut etre évalué à environ 1,20 $\mathrm{m}$ (fig. 34). C’est dians le voisinage immérliat de re vestige qu'ont été retrouvés 13 fragments de céramique attique à figures rouges du we siède, recollant avec certains autres découverts antérieurement notamment dans la partie sud-onest de la cour d'accueil. D'autres fragments des mèmes vases devaient etre ultérieurement découverts dans les couches cil et caz à l’intérieur du couloir prolongeant la porte monumentale. Leur regroupement a permis de reconstituer partiellement deux scyphos. Sur l'un d'entre eux, d'un style excellent, est representé d'une part un jeune enfant a cheval se dirigeant vers une jeune femme, un autre groupe etant constitué par la même femme et par un personnage barbu qui lui fait face. Ja femme semble des deux còtés prendre une altitude de refus. Cous supposons que ces scènes se rapportent au mythe de Poseidon Hippios et de ldéméter (fig. 35). L'autre, de style plus négligé, présente drux groupes de personnages affrontés, séparés par un autel, l'un des personnages tient un objet difficile a préciser. Il n'est pas interdit de pensere que ce vase soit une imitation italique de vase attique (figr. 36).

La partie sommitale du tas de pierres olservé a l'entrée de la cour comportait, an contact du mur, les vestiges d'un four presque rond, d'un diametre d'environ 1 m à l'extérieur, la dimension de la sole située à l'interieur etant de 0,50 m et l’épaisseur des parois de 0,25 m. Ce genre de four paraît adapté a la cuisson de la poterie fine. Ians le voisinage ont été trouvés des fragments de lécythes en céramique pseudo-ionienne tardive, présentant des traces de peinture rouge (fig. 37). Les formes de ces rases sont, en céramique attique, de la seconde moitió du ve sièrle, mais il n'est pas impossible d'admetlre qu'ils alemt été façonnés au tour, peints ot cuits sur la rolline saint-Marcel all cours du we siecle.

\section{L'enceinle, la porte monumenlale el le couloir.}

Yous avons déja ru. à propos de la date de lenceinte, que cette dernière avait été reconstruite à lat fin du we siècle de quau nord de la porte avait eté aménagé à l'intérieur du mur un nouveau chemin de ronde. Cn mur barrant la porte d'entrée a été observé dans 

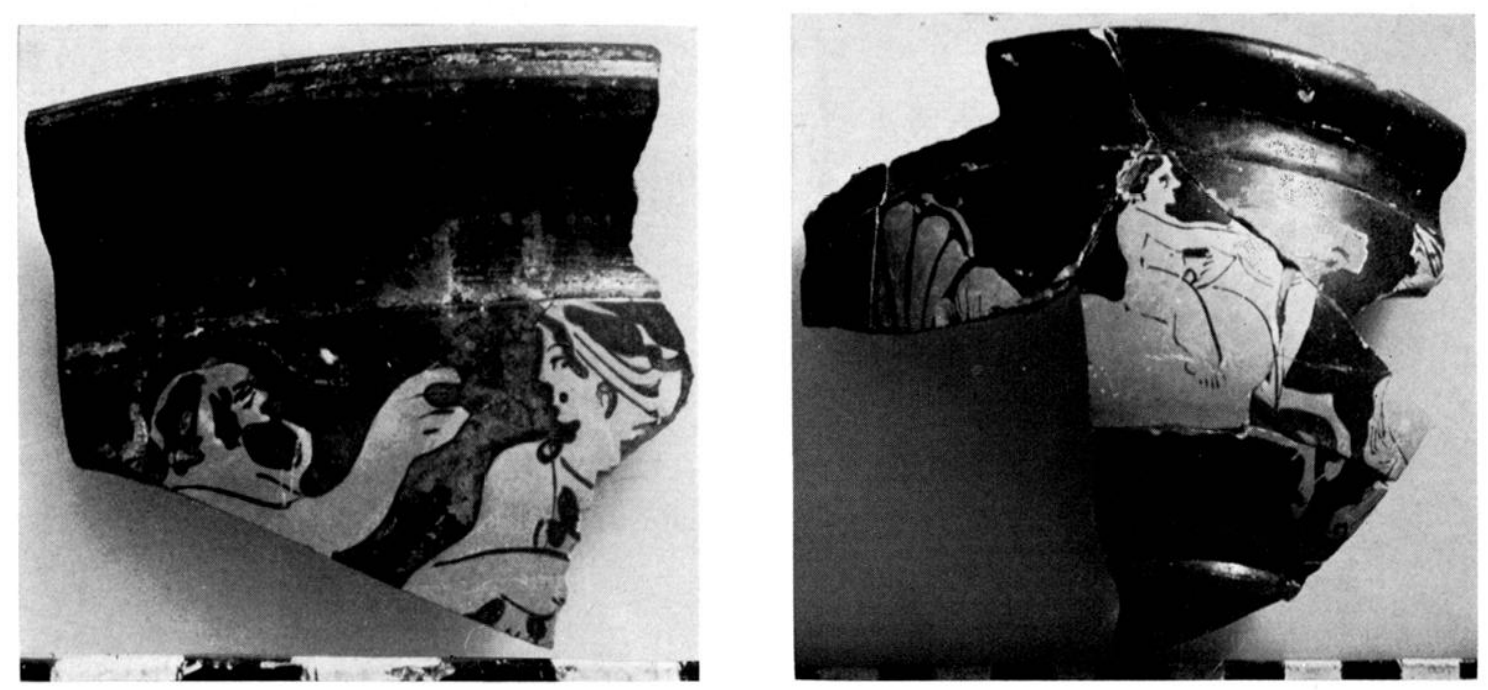

35 Fragments d'un scyphos attique a figures rouges decouvert dans la courder ca de la cour d'accuril.
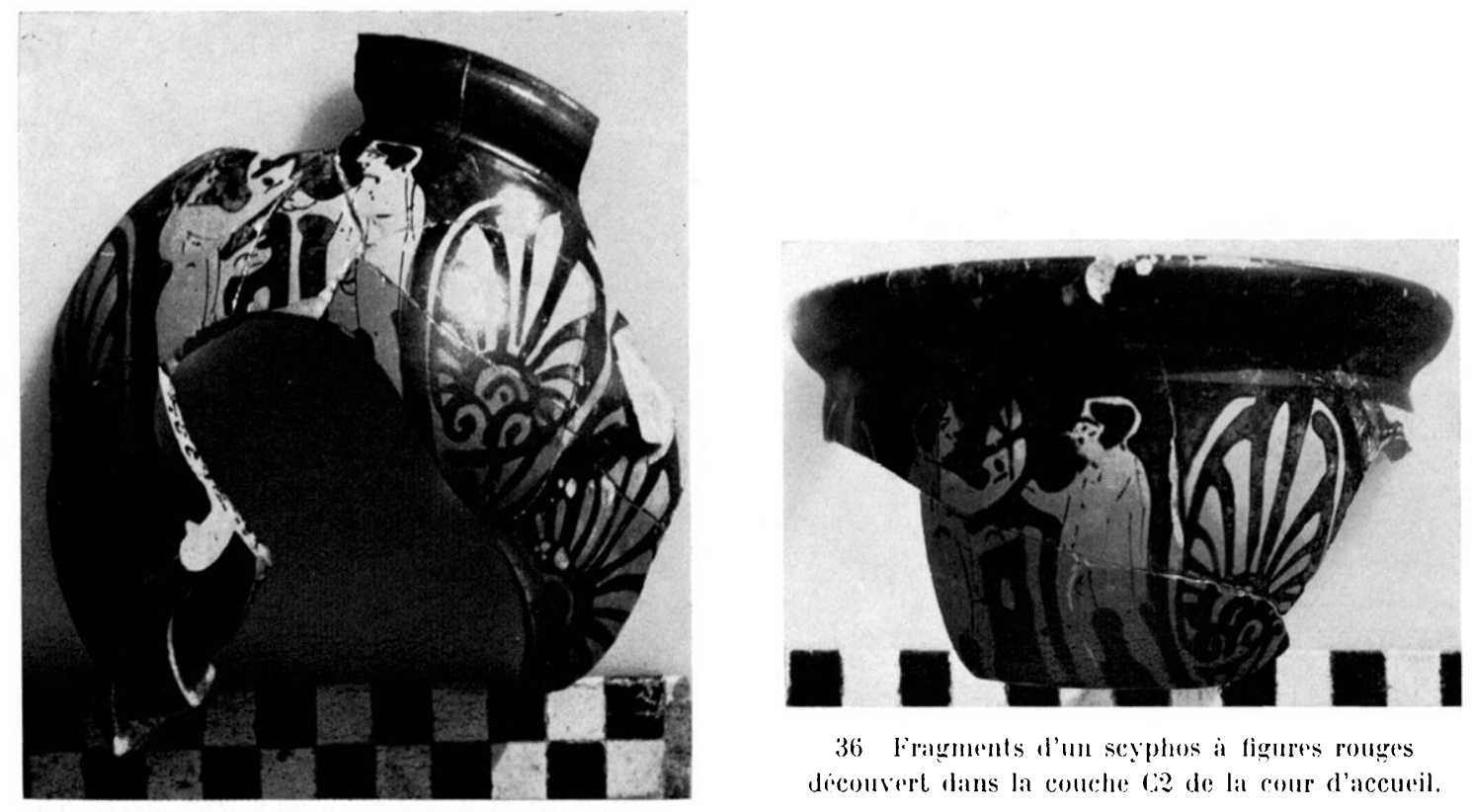

36 Fragments d'un scyphos à figures rouges decouvert dans la couche ca de la cour d'accueil.

les carrés 312 et $302 \%$. Il s'agit vraisemblablement d'un habitat de fortune aménagé an $w^{\text {e }}$ siècle, peut-être pour loger les potiers. Ce mur était posé sur le sol E1.

L'étude en stratigraphie des remblais comblant le passage de l'entrée monumentale sur une hauteur de $2,20 \mathrm{~m}$ (fig. 38 ) a mis en évidence plusieurs cour hes de remblai superposées.

Au-dessus de la couche li, de $-2,70$ m ì $-2,48$ m, un niveau de sable argileux dù au ruissellement dans le couloir après la période d'abandon consécutif à l'incendie du début du ve siècle. Ensuite, de - 2,30 a . 2 m une courhe Cas de cailloutis quasi-sterile a toutefois liver une fibule de La Téne Ia el une anse de coupe altique du ve siecle. Il s'agit d'une couche formée naturellement par la ruine des édifices voisins mais partiellement nivelée, soit en vue d'établir un passagre, soit pour des habitats de fortune. Ia seconde couche de remblai, de $-2 \mathrm{~m}$ a $-1,40 \mathrm{~m}$, notre couche C2. contient une tris 

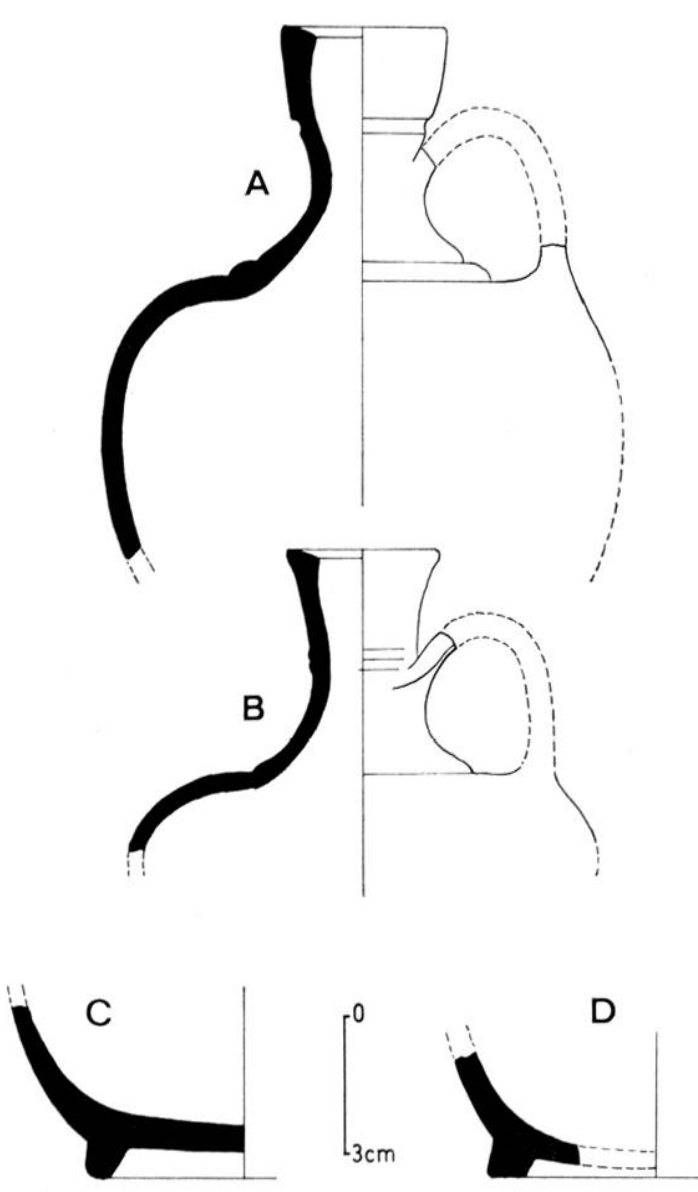

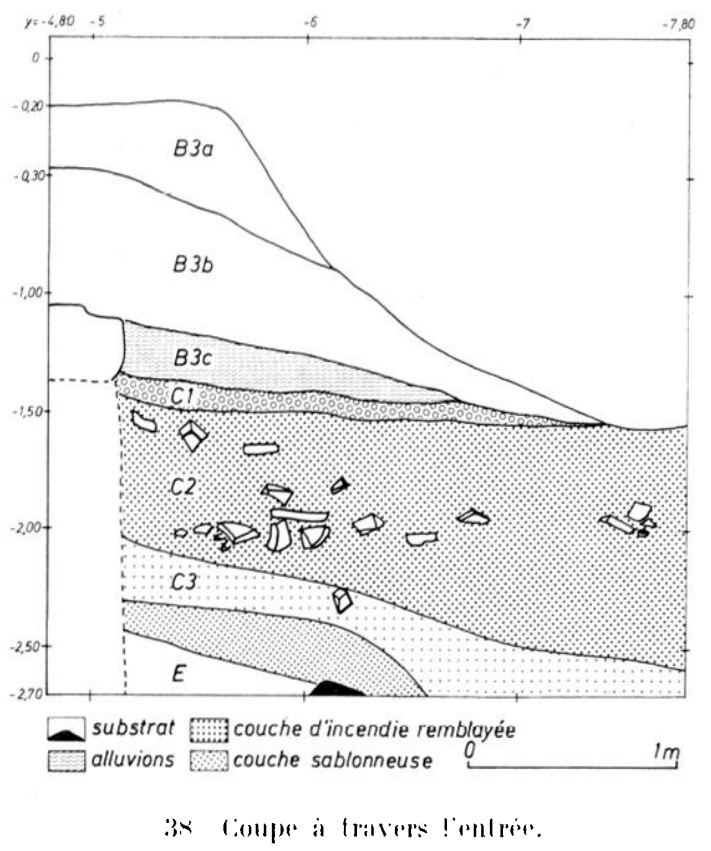

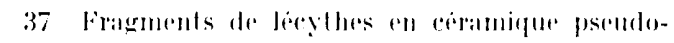

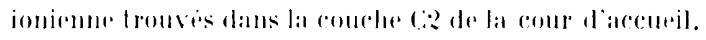

forte proportion de débris d'incendie provenant de la couche l). Elle est daté flar de la ceranique attique à figures rouges du $r^{\text {e }}$ siocle. Elle est rontemporaine de l'ulilisation de la rour d'accueil par des potiers. I a troisieme. Cl, de - 1,50 m à - 1,35) m. contenait de la céramique pregnathia ou attique tardive a palmettes de la fin du we sierle. C'est une couche de remblai contemporaine des réfections de la porte et de l'enceinte. Au-dessus de cette couche a eté aménagé un seuil en pierre. de $1,60 \mathrm{~m}$ a 1,0$)$ m ce seuil est constilué d'une stile en grès remployée. dans laquelle a été pratiquée une feuillure pour la porte el deux mortaises profondes pour le passage des jambages en bois fig. 39. A ce seuil correspond un sol amenage en pente, qui correspond a la courhe Bi3r de la coupe. Cetle rouche contenait de la céramique campanienne ancienne du II $^{\mathrm{e}}$ siecle. le seuil comme le sol manifesteraient done l'existence d'une période d'habitals sommairement aménagés, avec une porte en bois au cours du In $^{\mathrm{e}}$ siecle. Il s'agirait done dans notre stratigraphie chronologique d'une période Bi3r. Celte couche est contemporaine des fonds de cabanes de lal Tine Ir observes dans le couloir d'entrée

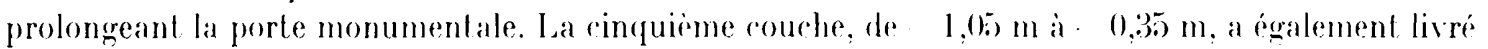
de la réramique campanienne, ainsi qu'un chenet votif gaulois à tâte de bélier. C'est notre couche B:3b du couloir, qui date de la Tène II. Elle marque véritablement le début de l'oppidum de la fin de La Tine, apris les traces tries sporadiques d'ocrupation de La Time le. l ne seconde couche, de La Tene II/La Tine 111 , de $0.47 \mathrm{~m}$ a $0.20 \mathrm{~m}$ correspond a not re courche Bi3a ifig. 38).

L'espace correspondant au couloir d'entrée prolongeant la porte monumentale ayant ate fres: fortement perturbé, surtout dans sa partie médiane, il a ete difficile d'en etablir la straligraphie. Toutefois une coupe pratiquée sur le bord sud, dans les carrés :30:3 et 304, en $x-21$ nous a permis les observations suivantes : nous avons pu distinguer en partant du bas, un lambeau de couche Ii 


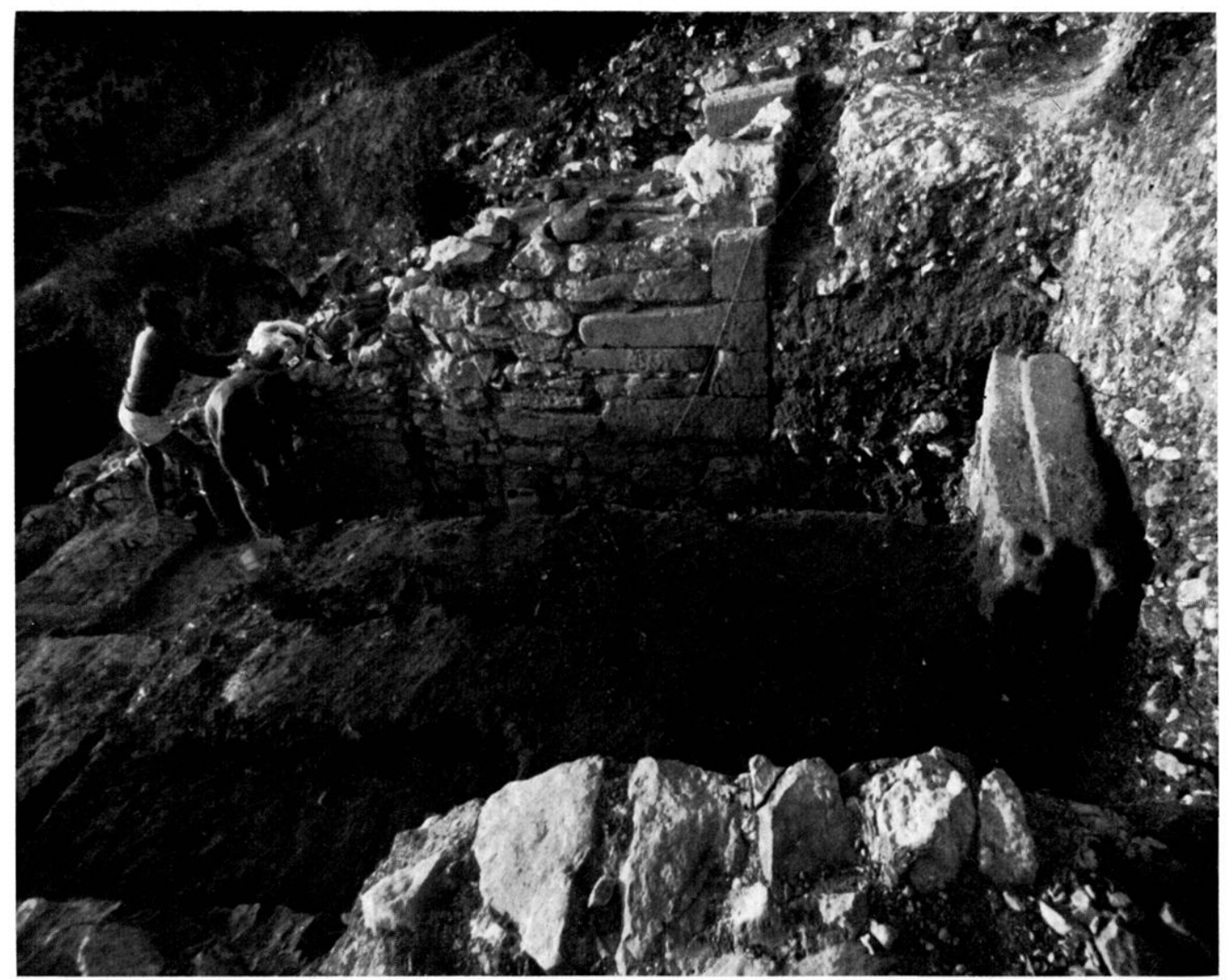

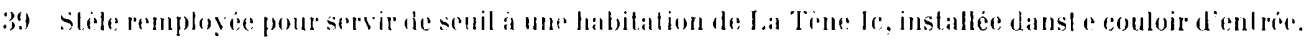

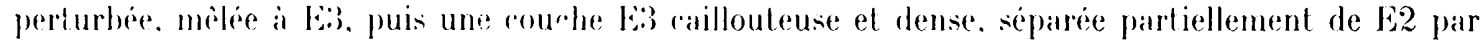
des lambeaux de sol en argile verdatre puis une couche C.) (omposé d'un cailloutis dense presque stérile. Ia courhe $F:$ et la rourhe $C_{3}$ ont livé des fibules hallstattiennes finales d'un style de

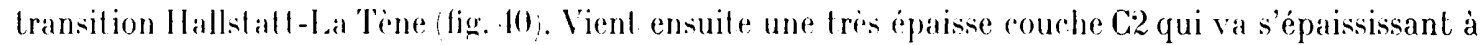
la fois vers la porte, en descendant. el vers le milieu du couloir. enfin une couche C1 d'abord de faible épaisseur, puis lres epaisse rers l'entré. C'est rette courhe qui domine dans tout le tiers nord-ouest du couloir. Elle est prestu'exclusivemenl composie du remblai de l'incendie du ve siecle, avec de rares tessons de reramique allique i figures rouges, el des fragments plus rares encore de céramique prégnathia. . Iu-dessus de rette couche, plusieurs niveaux de la Téne Ic a la T'ene III, trís difficiles a discerner dans les profondeurs, ou elles senfoncent dans les rourles C1 el Ciz. Ces couches nous ont toutefois livé des vestiges de sols et de constructions dates par une fibule de La Téne Ic (B3isc) el par une fibule de la Tine II (are seul ronserve) (B:3b) (fig. 40).

A partir de 322 les couches se distinguent mieux el correspondent aux installations de l'oppidum collique de la fin de lal Téne, que nous allons mainlenant étudier sommairement. 

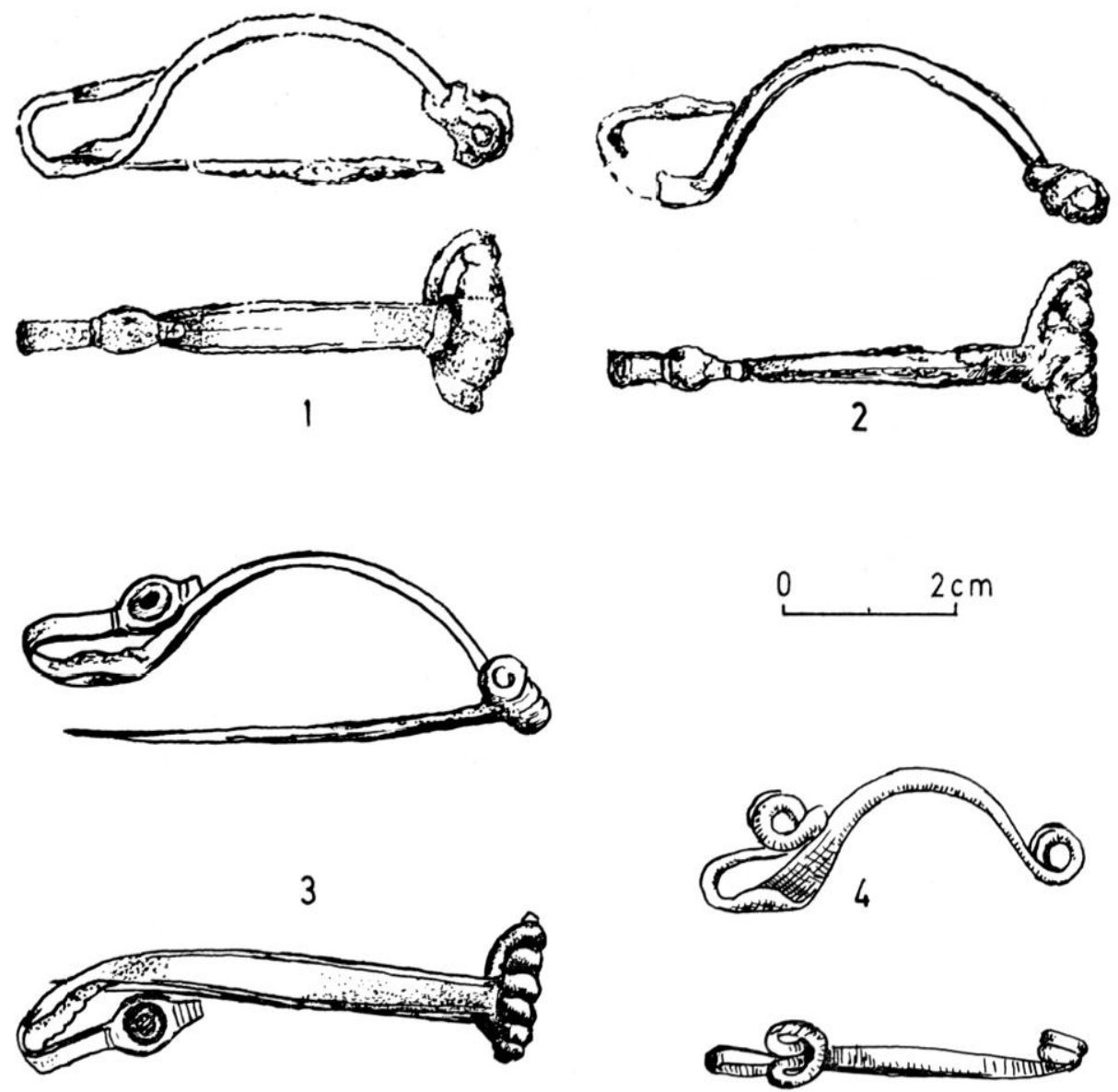

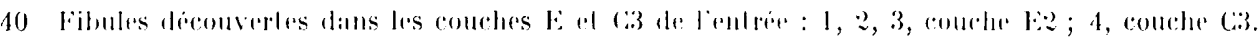

VII. Les inslallalions de l'oppidum gaulois depuis la fin du III se siècle al. .J.-C. jusqu'au début de notre ère (fig. 41).

Les vestiges relativement les mieux conservés ont été décourerts dans deux régions: au sondage 7 , à son extrémité méridionale, et en son milieu, restes de trois ansembles d'habitats et d'une conduite d'eau, au S. 11, dans sa partie sud-est, restes d'une maison de La Tène II, vestiges d'un quartier d'habitation romportant plusieurs cases, et vraisemblablement une écurie. Ces différents vestiges sont beaucoup moins complets et cohérents que ceux du sondage 8 , en raison de leur faible profondeur, et des travaux qui les ont en grande partie anéantis à l'époque romaine. C'est ainsi far exemple qu'au chantier central subsistait simplement un curieux blocage en pierre (fondation de citerne?), des restes de rigoles en pierres dressées, dans les carrés 134 at 141. at des restes de murs en pierres sieches: de lia Tène II et III.

Au sondage 7 il faut distinguer trois séries d'habitats :

1, les restes d'une case carrée de $4 \mathrm{~m}$ de coité située au sud-ouest de la conduite d'eau dans les carrés 108, 109, 98, 99, la conduite d'eau elle-mème étant située dans les carrés 97, 107;

2. des témoins de sols de La Tène II et III situés au nord-ouest de la conduite dans le carré 106; 


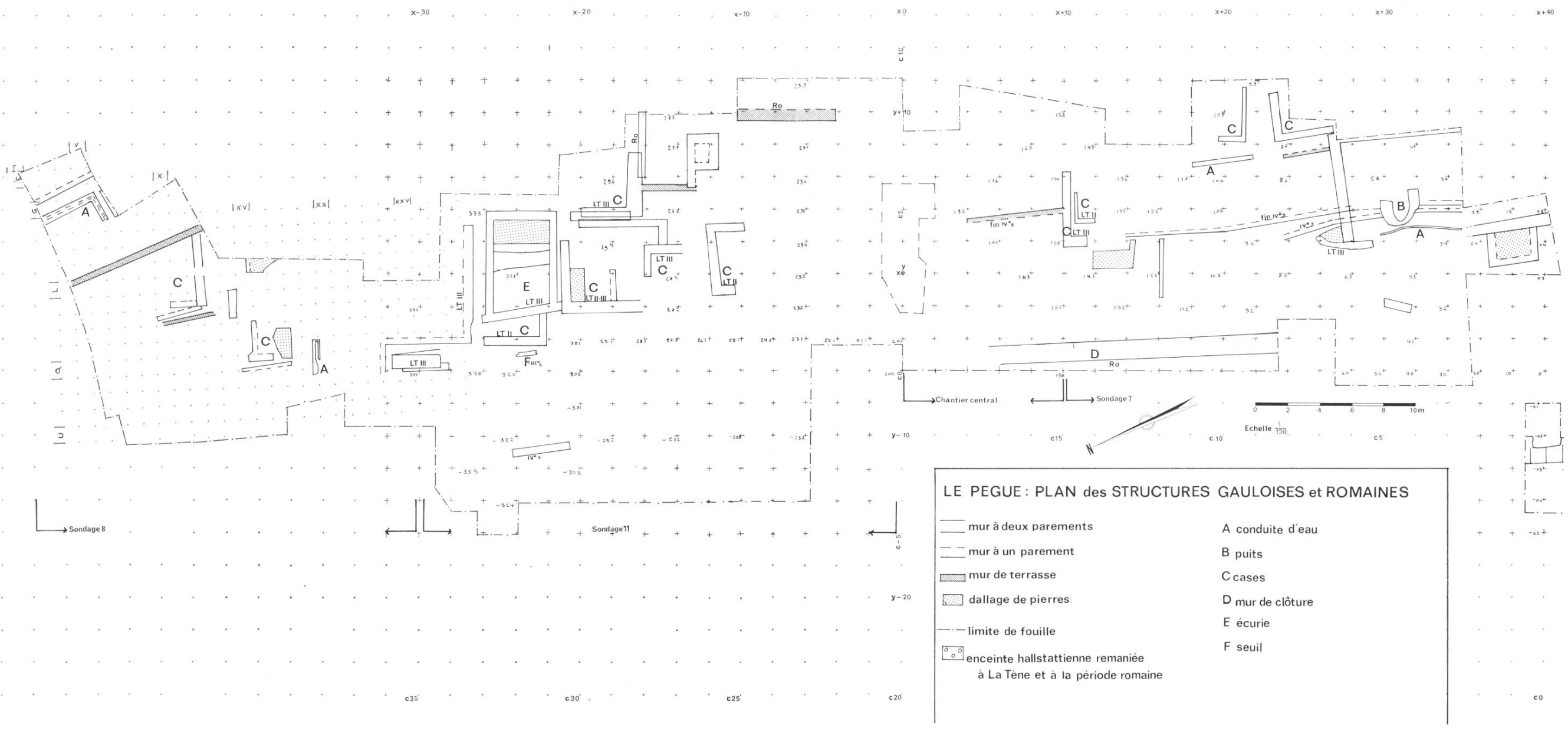

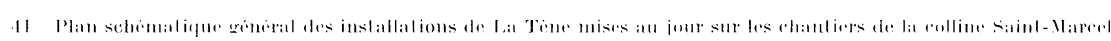




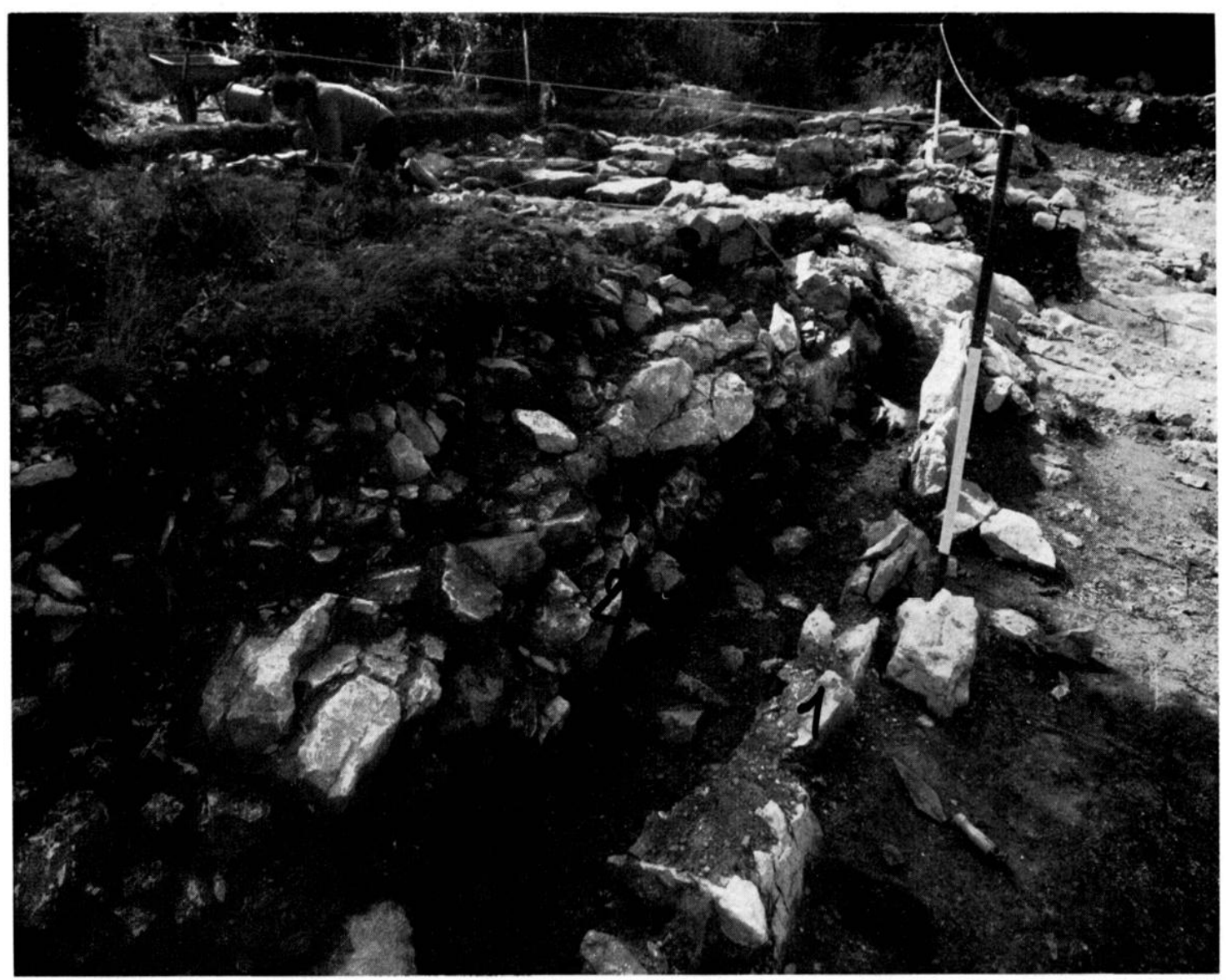

4: Vue de la comduite d'all de l.a Trime 111 .

3, un ensemble complexe de murs partiellement conserves de vestiges de fovers, d'un puits creusé dans le sol, d'une autre conduite d'eau coulant en sens inverse de la première. Ces différents éléments à cheval sur le mur hallstattien supérieur, et en partie appuyés sur le mur de terrasse médian de la fin du cre siecle, faisaient vraisemblablement partie d'une demeure relativement étendue ayant pris la place de la maison $\mathbf{A}$ de la période hallstaltienne.

Les deux premiers groupes sont orientes surdest nord-ouest et semblent avoir ete alimentés en eau par la conduite qui coulait du nord-nord-est au surl-sud-ouest. Lal grande demeure est orientée nord-est sud-ouest, et parait avoir été ravitaillée rn eau, d'une part par le puits, d'autre part par la seconde conduite d'eau, qui coulait du surl-ouest au nordest. La raison de cette difference tient a l'ensellement dans le substrat entre $x=30$ et $\mathrm{x}=34$. Au-delà de cet ensellement la pente remonte, d'où l'arrèt, de la conduite. Dans l'ensellement ontéte creusés en tout sept puits, pour atteindre la nappe d'rau qui s'y formait temporairement lors des phues. Ia seconde conduite d'eau venait de la partie sud de l'oppidum.

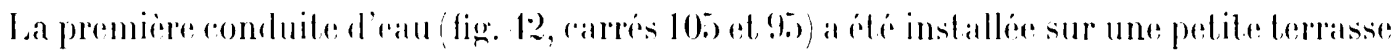
amenagee dont le sol etait constitue par un raillout is calcaire dame. Eille comportait une simple ligne de pierres plates plantées verticalement du ròté aval. Lal terrasse de la conduite 
reposait sur un mur de $0,40 \mathrm{~m}$ de hauteur au-dessus du sol de l'époque, elle était dominée vers l'amont par un autre mur de mème hauteur. Cé dispositif était situé à l'emplacement du mur hallstattien de terrasse supérieure, il n'était conservé que sur une longueur de $6 \mathrm{~m}$. Au pied du mur de terrasse supportant la conduite d'eciu a été trourée une perle de la Ténell. I)ans la conduite ont été recueillis des tessons campaniens et romains républicains, des débris d'amphores, une obole massaliote du rie s. av. J.-Ca, de la céramique peinte ganuloise et de la poterie vulgaire de La Tène 11I. La conduite se terminait dans le carré 97 par un déversoir aménagé avee des pierres lutées aver de l'argile. Elle remontait à la période de la Tène II. Il restait de son plus ancien étal, sous la terrasse finale, une série de pierres plates plantées sous-jacentes.

Au nord-est de la conduite a été trouvée, dans les carrés $108,1099,98,99$, , une case gauloise réduite à ses fondations sud-ouest et à des fragments de ses fondations nord-est et surl-est. Ses dimensions étaient de $4 \mathrm{~m}$ sur 4 . A l'intérieur de la case et dans son roisinage immédiat ont pu être distingués trois niveaux. La couche B1 appartenait entièrement à la période gallo-romaine précoce. Cous avons dégagé un sol rorrespondant à cette époque dans le carré 109. Ce sol se retrouve de l'autre coté clu mur et se soude à un autre mur du rarré 88 appartenant au second groupe d'habitat. La couche 132 se trouve à environ $0,20 \mathrm{~m}$ au-dessous de B1, la couche B:3 également à $0,20 \mathrm{~m}$ au-dessous de Bi2. La courhe B1 rontrnait deux monnaies de Marseille, une pendeloque en bronze de La Tène III, des fragments de céramique (ampanienne (formes j) et 31 ), des morearux de céramique gauloise peinte. de dolium à pâte rouge et à gros dégraissant. Elle contenait également de la poterie galloromaine précoce et même de la terre sigillée italique. L'utilisation de la conduite semble done s'être poursuivie jusqu'au début de notre ère.

La couche B:2 contenait l'extrémite de laare et le ressort d'une fibule de la Tène IIJ, des fragments de céramique campanienne A, un morceau de céramique indigène imitant la campanienne à palmettes, des fragments de poterie peinte galuloise, un morceau de perle de verre à décor oculé, un col d'amphore républicain. La rouche B3, se terminant triss vite sur une remontée dlu substrat, n’a rien fourni d'intéressant.

Dans le carré 106 la couche li2 contenait les traces d'un foyer, daté par un fond de coupe campanienne à bande blanche, un fragment de fibule du type de Nauheim découvert en $x=18,20, y=6,80$, et par des tessons de campanienne à bande blanche se regroupant, sur lesquek avait été inscrit un graffite en lettres grecques: OYIP(OXIAC. A proximité de ce foyer, dans la couche de remblai l31 a été découvert au point $x=18,30, y=5,30$, un fragment de calotte cranienne humaine.

Sous le sol P2, séparé de lui par un remblai de $0,30 \mathrm{~m}$. nous arons trouvé un second sol durci par la chaleur. La couche B33 en rapport avere niveau est datée par un morean de fibule en fer de La Tène. II trourée dans le carré 107 et par une obole massaliote du milieu du II $^{\mathrm{e}}$ siècle av. J.-(C. Sous le niveau B33 a été trousé un autre témoin de sol en argile durci par la chaleur, appartenant à un niveau plus ancien, peut-être du Ir $^{\mathrm{e}}$ siècle.

Le groupe d'hathitations du surl-ourst comprend des murs en pierres sèches formant angle droit, et se rattachant au mur de terrasse de lat Tene final dominant le mur de terrasse

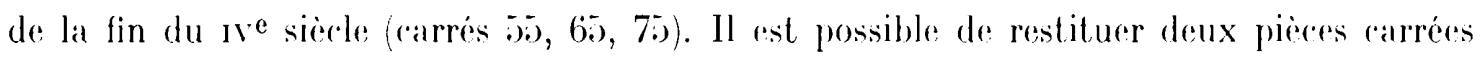


attenantes de $4 \mathrm{~m}$ sur $3 \mathrm{~m}$. A $2 \mathrm{~m}$ au sud-ouest de celle de deux pièces qui est située le plus au nord-ouest, un emplacement de puits creusé dans l'ensellement rocheux a été entouré d'une murette en pierres sèches de 2 m de diamètre. Ciette murette etait sans doute la base d'une voùte légère en branchages et torchis protégeant le puits. Le puits et son entourage sont à cheral sur le mur de terrasse de la fin du re sièrle, qui a été détruit à leur emplarement (carrés 45) 46). Le puits lui-même présentait un diamètre de $0,80 \mathrm{~m}$, il était taillé très régulièrement jusqu'à une profondeur de $0.80 \mathrm{~m}$ sous le niveau de l'époque. Dans les (arrés 25. 35), 45) ont été trouvés les restes d'une conduite d'eau, sous la forme d'une ligne de pierres plates plantées. La plupart des puits qui ont été repérés et vidés contenaient, à l'exception de celui du carré 4.), un remplissage médiéval. Le puits des carrés 57, is était rempli de cendres de bois et de tessons médiévaux, aver lesquels a pu être reconstitué un rase ponté actuellement en dépôt au centre de recherches médiévales de l'Lniversité d'Aix (Jlle d'Archimbault).

Dans les carrés 65 et 75, perpendiculairement au mur romain, mis au jour en 1962, nous avons trouve à un niveau inférieur (B3) les restes d'un mur de terrasse contre lequel avait été installé un foyer. Te l'autre côté du mur romain nous avons observé, dans le carré 55, une calotte crânienue humaine à l'intérieur de la couche de remblai B3. Au sud-ouest de la calotte crânienne ont été trouvés dans la couche 333 des tessons de céramique campanienne, de céramique gauloise peinte et de céramique grise ampuritaine. Dans le arré 77 existait sous le mur de La Tène III les vestiges d'une fondation plus ancienne, également de La Tène III (B:2), prouvant que la demeure existait déjà auparavant à cet emplacement mais que son quadrillage était différent. A cet endroit, dans la couche B2, a été mise au jour une fibule de La Tène III en argent d'un type relativement ancien (fig. 43).

Dans le même carré, deux témoins de sol en terre durcie par la chaleur ont été découverts. L'un appartenait à la couche B'2, l'autre à une couche plus ancienne. A cet endroit. dans la couche 13:3 ont été trouvées sept oboles marseillaises du ri siècle ar. J.-C:

La calotte crânienne du carré a) a été trouvée à proximité d'un foyer plus ancien que la couche qui le contient et non loin d'un dispositif de pierres de support, à proximité duquel se trouvait un fragment de meule rotative en basalte. le puits se trouvait dans le carré voisin. Cet ensemble correspond à la cuisine et au foyer domestique. Yous connaissons maintenant, en ce qui nous concerne, et abstraction faite du sondage 8 et de son extension vers le sondage 11, cinq exemples de trouvailles de restes humains dans les couches de La Tène II-III, la plupart du temps dans des remblais à proximité de foyers :

1. à l'intérieur du carré 106 , a proximité d'un foyer de La Tẹne III, un fragment de calotte. rànienne ;

2 , dans le carré 5ij, pris d'un foyer B3,3, à l'intérieur d'un remblai 132 ;

3, dans le carré 67, à proximité d'un foyer de La Tène III, une vertèbre, des dents humaines et un couleau de lat Tíne I ;

4, dans une fosse siluée a proximité de foyers de La Téne II non loin de l'enceinte du village de plaine (fouilles de l'école);

5), au sondage 11, dans le carré 285, un maxillaire humain trouvé dans une conduite d'eau à proximité d'un foyer. 

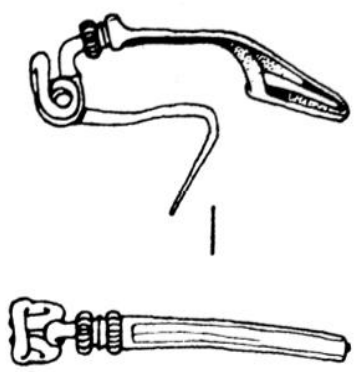

$0 \quad 2 \mathrm{~cm}$

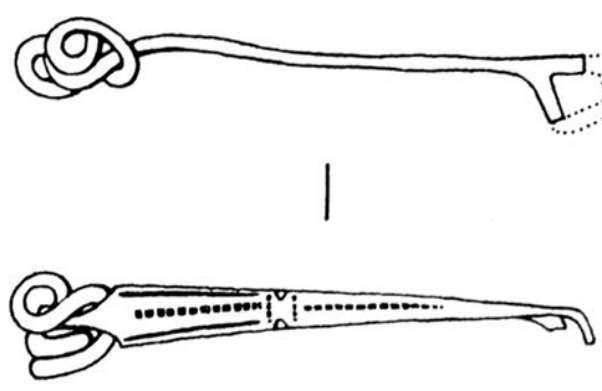

1:) Fibules de lai Time III trouvines press de la conduile diatu.
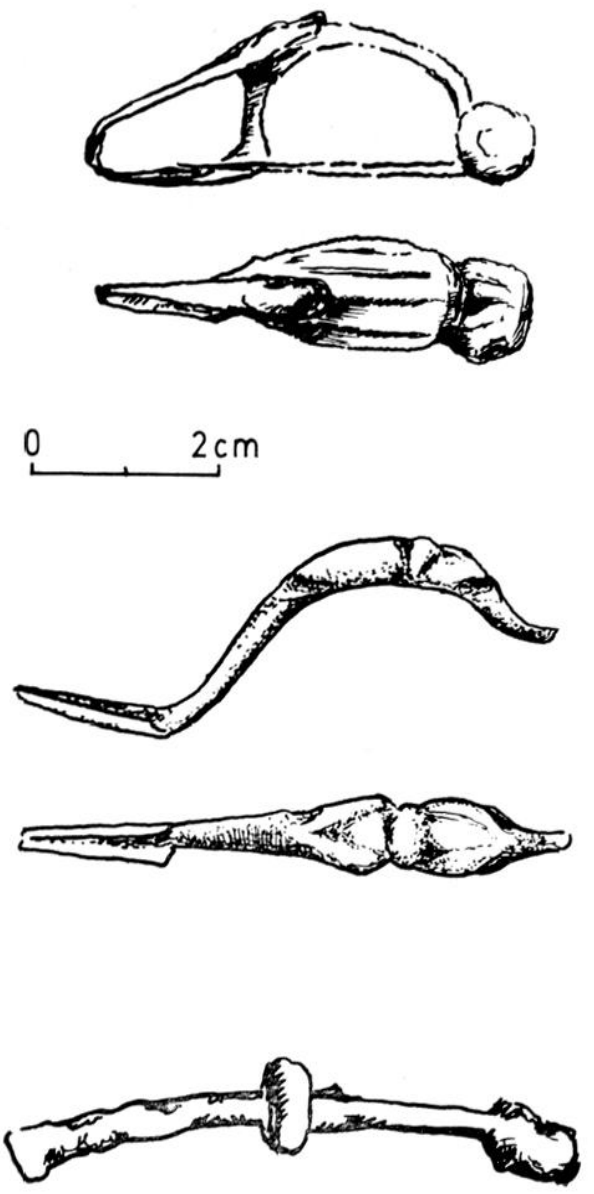

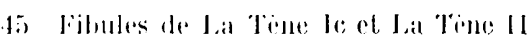
trouries an sondiare 11.

VIII. Les vestiges de La Tène Ic, La Tène II el La Tène $I I I$ du sondage 11.

Au-dessus de la tour intérieure, dans les carrés 20.3, 254, 25), 26:3, 26.1, 26.) ont été mis au jour les restes d'une case de La Tène II, datée par de la céramique campanienne ancienne. C'est malheureusement le seul vestige cohérent de cette époque, la plupart des murs de cette période ayant été soit détruits soit réutilisés par les constructions postérieures.

Si nous nous référons à la coupe stratigraphique pratiquée du nord-ouest au sud-est à travers la cour d'accueil, nous constatons que le terrain assez chaotique laissé par les ruines des fours de potiers du $\mathrm{I}^{\mathrm{e}}$ siècle a été systématiquement remblayé à la lin du re siècle, puis ce dernier niveau a été à son tour, au cours des III $^{\mathrm{e}}$, $\mathrm{II}^{\mathrm{e}}$ et I $^{\mathrm{er}}$ sièrles tantôt surhaussé tantôt creusé à nouveau par les générations successives de cabanes. En plan, plusieurs murs et installations de La Tène III ont été dégagés au dessus de la cour. Ils se répartissent en trois phases : une phase gallo-romaine préecere, une phase La Tène III finale, une phase la Tène. III plus ancienne.

1, A la phase gallo-romaine précoce se rattachent les murs fiaisant angle droit des carrés 278 . $286,285,295), 296$, ainsi que les remaniements supérieurs dans les murs des carrés 275, 276, ces 


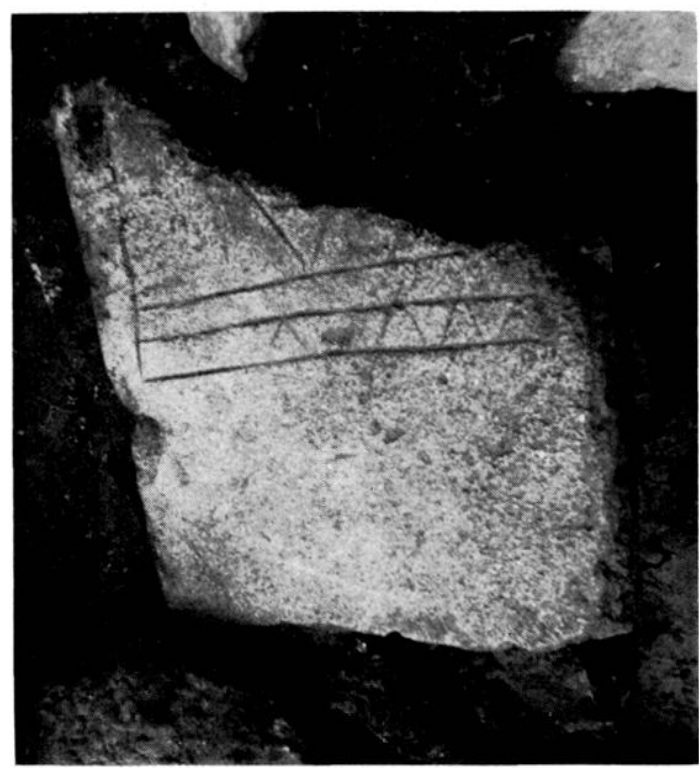

14 stile gravin, remployie

dans un mur de Ia Tine III all sondage 11 .

derniers datant d'une période plus ancienne Bla. Aux murs de la période gallo-romaine précoce (B1a) est atlaché un sol en cailloutis calcaire très fin, damé, doni le niveau est compris entre $0,95 \mathrm{~m}$ et $0,90 \mathrm{~m}$ au-dessus du point origine, $1,50 \mathrm{~m}$ sur la terrasse supérieure (carrés $266,267,276,277,286$, $287)$.

2, A la période finale de I a Tène III appartiennent d'une part un sol trouvé sous le sol Bla et. qui se raccorde aux murs réutilisés pendant la période gallo-romaine précoce dans les carrés :302, 303, 304, 292. 29:3, 282, 28:3, el d'autre part des murs datant de la période de Ia Téne finale mais abandonnés par la suite dans les carrés 302, 303,3, 304, 292, 293. 282. 28:3, ainsi que des éléments de dallage se rapportant á ces derniers. Le sol B1b est séparé du sol Bla qui le domine par une couche d'une vingtaine de centimetres de cailloutis damé. Il est situé, sur la terrasse inférieure à une altitude variant entre $0,71 \mathrm{~m}$ et $0,68 \mathrm{~m}$, sur la terrasse supérieure a $1,30 \mathrm{~m}$. Lans le carré 285 , a l'altilude de $0,68 \mathrm{~m}$ et au point $\mathrm{x}=-16, \mathrm{y}=3.102$ a été découvert un maxillaire humain complet pris dans le remblai B1b, à proximité d'un foyer. En effet, dans le carré 27\% deux pierres plantées délimitaient avec l'angle du mur un emplacement de $0,30 \mathrm{~m}$ sur $0,60 \mathrm{~m}$ qui était un fover construit. Encore une preuve de cet usage qu'avaient les Gaulois de La Tine II-Ill de déposer des reliques humaines à proximilé des foyers domestiques. Dans les carrés 304 et 305 a été observé, à l'intérieur de la case, un dispositif consistant en une sorte de conduite installee avec des tessons, aboutissant a ce qui n'était plus qu'un amas de pierres disloquées. mais qui pourrait fort bien correspondre à un évier primitif. Il apparait d'autre part dans tout re quartier que les espaces entre les cases ont été régulierement utilisés pour canaliser les eaux de ruissellement el pour les recueillir. I cheval sur les carrés 30:3 et :304 a été découverte en remploi dans une fondation une stèles gravée d'époque hallstattienne ou de Ia Tène ancienne. décorée d'un losangre et d'une bordure inférieure de triangles. Ia stèle est incomplete (figr. 44).

A la mòme période (B1b. La Tène tinale) appartient un angle de murs découvert dans le (arré 275 se raltachant à une réfection tardice du mur hallstattien de la cour d'accueil. Ces constructions de I a Tëne III finale se poursuivent dans les carrés $266,267$.

3, Au sol B2 (début de Ia Téne III) se rallache un mur sous-jacent au mur B1 découvert dans les carrés 282, 292 et 302, ainsi qu'un dallage conservé dans les carrés 302, 303, un angle de murs et un sol damé en argile dans les carrés 27.3, 274. une partie de murs et un foyer construit dans les carrés 285, 29\%. 
4, La période 133 est représentée par des traces de foyers et des restes de murs sous-jacents aux murs 132 et 131 dans les carrés 293, 283, 273, 274. Le foyer du carré 273 est à l'altitude de $+0.39 \mathrm{~m}$. Dans le carré :304 est apparue une rigole en pierres plates présentant une forte pente. correspondant au niveau B3.). Au mème emplacement avait été auparavant observée une autre rigole superposée au niveau B2 et située entre deux cases de La Tène III. La rigole appartenant à la période de La Téne II (B:3) aboutit à un emplacement où ont été mis au jour de nombreux fragments de dolium. Il s'agit d'un dispositif destiné à recueillir les eaux venues d'une conduite supérieure.

\section{Les vestiges de La Tène au-dessus du couloir d'entrée el dans la partie inlermédiaire entre le} sondage 8 el le sondage 11.

Au-dessus du couloir d'entrée nous avons relevé dans les carrés 3201 à 325, 311 à 315. 301 à 305, les vestiges bien conservés d'un bâtiment rectangulaire de 5 m de largeur sur $7 \mathrm{~m}$ de longueur. Ce bâtiment comportait dans son tiers sud-est un dallage assez massif (carrés 305, 315, 325), puis une rigole aménagée également aver des dalles, enfin, un sol très épais en argile (carrés 304, 314, 324). Yous avons supposé qu'il s'agissait d'une écurie. Construit all cours de la période de La Tỏne II (B3ia), ce bâtiment était encore ch usage au cours de la période de La Tène. III. Son sol se raceorlait à la couche de la fin de la T'ène Il (Bi3a) (fig. 4)).

Sous re batiment nous avons mis au jour des dallages partiellement conservés (carrés 314, 324, 334, 313, 323, 333), ainsi que les trois murs d'une case gauloise (311, 321, 3331). Ces vestiges appartiennent au début de La Tène II (couche 133b). L'un des murs est daté par un fragment de fibule de cette époque. La case de la t'ene 11 se poursuivait vraisemblablement vers le nord. au-dessus de la porte. Sous ces vestiges de murs, dans les carrés 3333 a 330, 3:23 à 320, 31:3 a 303, nous avons observé la présence de quelques dalles éparses, de témoins de sols, de vestiges de foyers inclus dans la couche Bi3e et remontant à la période. de La Tène le, comme la trouvaille dans cette couche d'une fibule de cette période l'a prouvé (fig. 45).

Dans la partie intermédiaire entre le sondage 8 et le sondage 11, confiée à Ch. Lagrand, nous avons trouré, dans la partie sud-ouest. et dans la paroi de la coupe, des restes importants de murs de La Téne III (131 et B32) dans les carrés 335), 334, 332, ainsi qu'un dispositif profond de drainage dans les carrés 331, 241. 331, 461. Lal découverte au sud-est de ce dispositif d'une cave profonde de La Tène III permet d'expliquer cet aménagement : c'était pour proteger la cave du ruissellement des eaux d'orage. C'est la première fois que nous trouvons une are de la Tène aussi bien conservée at aussi profonde.

\section{$\therefore$}

La première publication de Ch. Lagrand et J.-P. Thalmann portait uniquement sur le sondage 8 qui a toujours gardé sa pleine autonomie comme chantier de fouille personnel de Ch. Lagrand. Le grand mérite de ce travail précis, soigné. méticuleux a été de donner les bases solides d'une chronologie et un précieux fil directeur pour la compréhension de stratigraphies complexes at souvent très décevantes, puisque la plupart des couches les 
composant étaient constituées presque uniquement de remblais. 11 m'appartenait de faire une première publication provisoire des fouilles beaucoup plus amples qui, à partir de 1962, ont mis au jour au sondage 7 des restes dhabitations plus vastes, puis, à partir de 1967. au sondage 11 les vestiges beaucoup plus monumentaux et spectaculaires d'un systemes fortifié, sans omettre celles qui, pratiquées, entre 1961 et 1963). près de l'école du Pègue, ont mis au jour le village fortifie lui-mème, dans la plaine de l'Auzon. Ces fouilles ont, grâce à l'analyse des vestiges monumentaux rombinée à l'étude des sols et des couches strationraphiques, permis de confirmer et de préciser less résultats déjà obtenus en ce qui concerne les périodes d'orcupation de l'oppidum :

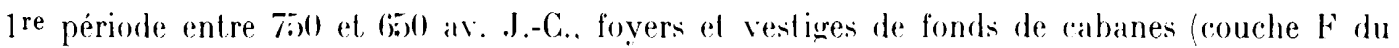
sondage 7 );

2 e période. de 525) at 480. installation sur la colline d'un oppidum fortifié. Trois phases de constructions: E3. enceinte porte. premier état de la tour bastion intérieure au $\$ .11$. premier état des habitations du S.7; F.2, au s.11, aménagement d'une porte en bois dans l'entrée. construction de la cour d'accueil, deuxième état de la tour intérieure. aver double plateforme de tir ; $\$ .7$, deuxième état des habitations. El. désaflectation de la double plateforme du bastion. La Iroisieme phase a été suivie, entre 490 et 480 as. J.-C.. d'une période d'abandon de l'oppidum ;

3e période, installation d'un dépót de grains sur la colline vers 480 av. J.-G. Incendie de ce dépòt ;

4e période, vers 400, habitats de fortune installés par les envahisseurs celtiques dans les ruines. Fond de calane au S.11 dans l'escalier de la tour, indices d'occupation ou de passage dans le couloir d'entrée, ainsi qu'au $\$ .7$;

¿e période, installation, au cours du $v^{\text {e }}$ siercle, d'ateliers d'artisans, notamment de potiers, au S.11, fours de potiers dans la cour d'accueil. Au s.7. mur de terrasse et murs d'habitations remaniés ;

fie période, à la fin du $\mathrm{s}^{\mathrm{e}}$ siècle, grands travaux de reconstruction de l'enceinte el de la porte, remaniements profonds dans le systime de terrasses. Ces travaux n'aboutissent pas à un établissement durable. S.11, indices de reconstruction de l'enceinte. de la porte et du chemin de ronde intérieur, bouleversements dans le corridor d'entrée; $\$ .7$ mur de terrasse ;

7e période, à la fin du me siecle av. J.-C.. squalterisation progressive des ruines : s.11, vestiges de fonds de cabanes, sol el seuil dans le couloir d'entrée. au $\$ .7$. sols el vestiges de fovers ;

se période, au débul du ne siècle. installation d'un oppidum gaulois sur la colline. élablissement. d'un systime compliqué de gouttieres et de conduites d'eatu. succession d'habitats trés modestes, installés de faģon plus ou moins anarchique depuis le début de La Téne II jusqu'à la période galloromaine précoce (début de l'ìre chrélienne). s.7 et s.11, groupes d'habitations juxtaposées el souvent. superposées, restes de murs el de foyers. Plusieurs phases à distinguer, après la phase Bi3c de la fin du II $^{\mathrm{e}}$ siècle: B3:3b, début de La Téne II : B33a, fin de La Téne II. début de La Téne III ; B2, fin de La Tène II, débul de Ia Téne III ; BI, fin de La Tène III, gallo-romain précore.

De cet ensemble de données se dégage une impression assez paradoxale, qui est celle d'une série d'expériences d'urbanisme plus ou moins avortés : avortée, la première expérience urbanistique, à la greeque, qui a a prine dure trente ans, et qui s'est terminée par une décarlence et une barbarisation. suivie d'abandon total. Arortée plus encore la seconde expérience de la fin du ve siècle, qui n'aboutit à aucune installation durable. Ce. n'est qu'à partir de la fin du zu e siècle qu'apparait, de facon d'abord timide et sporadique, un urbanisme spontané, a la gauloise. en cléfinitive beaucoup plus inorganique, beaucoup 
plus barbare que l'éphémère urbanisation de la fin du vie siècle. Et cet état de choses se poursuivara dans la Provence romanisée très longtemps aur cours des ${ }_{1} \mathrm{e}$ et $1^{\text {er }}$ siècles ar. J.-C.e, voire même jusqu'à la fin du règne d'Auguste.

Il n'est pas possible dians ce simple rapport d'aborder tous les problèmes que posent artuellement les fouilles du P’èque et l'énorme matériel qu'elles ont produit. Constatons simplement que ce site est d'une importance rapitale pour les donncés nouvelles qu'il nous apporte concernant :

1, la constitution des rivilisations régionales du Hallstatt final of leurs affinites;

2 , les influeners helléniques et lo commerce aves la Véditerranée des populations préceltiques et celtiques du vie a nII $^{\mathrm{e}}$ siècle ar. J.--C. ;

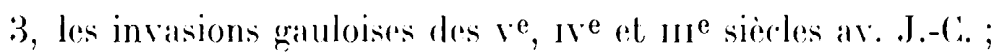

4, la formation de la civilisation de La 'Tene et la part qu'ont pu y prendre les peuples le la vallée du Rhòne comme intermédiaires obligés pour la transmission des éléments grees et etrusques;

5) les contacts entre liaulois envalisseurs et populations autochtones au cours des

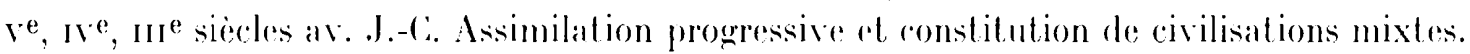

En tout etat de ause, les solutions que nous proposerions, les hypotheses que nous avancerions seraient toujours sujettes à révision, aussi bien en fonction des progrès ultérieurs des fouilles at des recherches que d'une analyse approfondie des matériaux'.

$$
\text { Jean-Jateques HatT. }
$$

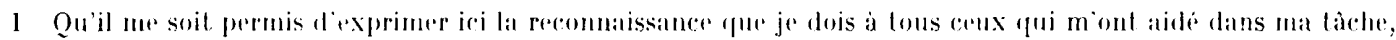

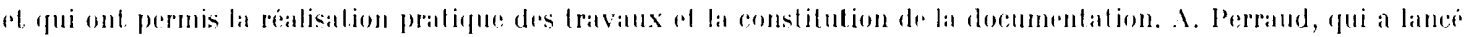

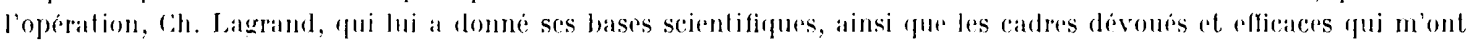

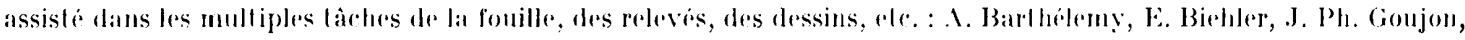

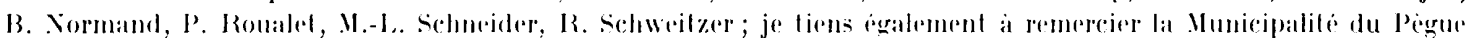

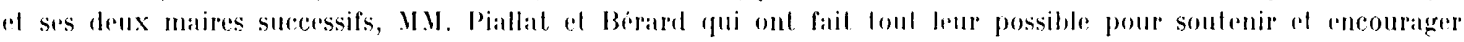

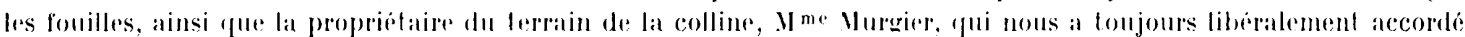

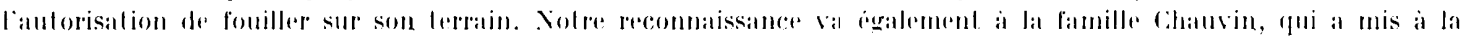

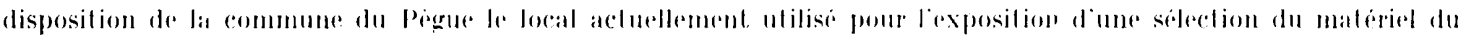
deport le fouilles. 